\title{
An extreme case of metabolic syndrome in a child; a multidisciplinary approach with bariatric surgery
}

\author{
Kariyawasam JP ${ }^{1}$, Liyanarachchi ND $^{2}$, Gunathilaka $\mathbf{G}^{3}$, Arambage $\mathbf{S}^{2}$, Wijeratne WGDTK ${ }^{3}$ \\ ${ }^{1}$ University Paediatric Unit, ${ }^{2}$ Paediatric Pulmonology Unit, Teaching Hospital Karapitiya, Galle, Sri Lanka. \\ ${ }^{3}$ University Surgical Unit, Colombo South Teaching Hospital, Kalubowila, Sri Lanka.
}

\author{
Correspondence: Dr. J. Prathibha Kariyawasam \\ e-mail: prathibhaka85@gmail.com \\ (D) https://orcid.org/0000-0002-7404-8264 \\ Dr. Nayana Liyanarachchi \\ e-mail: liyanarachchiuk@yahoo.co.uk \\ https://orcid.org/0000-0003-3920-8653 \\ Submitted on 29.08.2021 and accepted for publication on 23.12.2021
}

\section{Introduction}

Metabolic syndrome (MS) is a multifactorial disease associated with abdominal obesity, insulin resistance, hypertension, and dyslipidaemia. It is a known association with obesity due to insulin resistance (1). The diagnosis of metabolic syndrome is made if two or more of; fasting hyperglycaemia, hypertension, and dyslipidaemia are present in a child with obesity (2).

The rapidly rising prevalence of childhood obesity is related to the increased risk of MS. National Health and Nutrition Examination Survey (NHANES) data from 1988 - 1994 has shown a $4 \%$ prevalence of MS in adolescents aged $12-19$, which increased to $29 \%$ if limited to obese adolescents (3). Moreover, NHANES data from 1999 - 2002 showed MS prevalence of $7.8 \%$ in overweight and $44 \%$ in obese adolescents (4). Obesity prevalence among 5- to 18-year-olds in urban Sri Lanka is reported as $10.3 \%$ in 2016 (5). As it is increasingly prevalent with many complications affecting the quality of life such as cardiovascular events, type II diabetes, obstructive sleep apnoea, fatty liver, polycystic ovarian syndrome and psychological issues which includes bullying by peers, negative body image affecting child's personality, we need newer approaches to benefit patients' overall wellbeing.

\section{Case presentation}

A 13-year-old obese girl was admitted to the intensive care unit with aspiration pneumonia associated with apnoea during sleep. She was diagnosed with obstructive sleep apnoea syndrome (OSAS) complicated with pulmonary hypertension. She had hypoxia affecting her routine daily activities; thus, she was started on Bilevel positive airway pressure (Bi-PAP, with an expiratory/ inspiratory pressure; $12 / 5 \mathrm{~cm} \mathrm{H}_{2} \mathrm{O}$ ) and ambulatory oxygen therapy. She also had dyslipidaemia, impaired glucose tolerance, grade II fatty liver and hypertension.

This girl is the firstborn to healthy nonconsanguineous parents with a healthy younger female sibling. Her birth weight was $3.25 \mathrm{~kg}$, and she showed excessive weight gain from eight months onwards but showed significant weight gain with excess food intake over the last year, which was managed in the private sector with no improvement. She had increased appetite throughout, and diabetic control could not be achieved despite behavioural modifications and counselling by endocrinologists, paediatricians, and nutritionists. She continued to fight for food, and her friends were helpers for her at school. From 6 years onwards, she was treated for attention deficit hyperactive disorder with methylphenidate, 
and her food craving behaviour continued. With time, she started fighting with her sister. Later she developed features of depression and had even suicidal attempts. As sibling rivalry and depression continued to affect her quality of life, she was started on risperidone $2 \mathrm{mg}$ nocte and sertraline 150 nocte, at 11 years. She was on atorvastatin $20 \mathrm{mg}$ daily, metformin $2 \mathrm{~g}$ twice a day, and Losartan $25 \mathrm{mg}$ bd, for MS and its complications.

On admission to the intensive care unit with sleep apnoea, her weight was $88 \mathrm{~kg}$, and her BMI was $41 \mathrm{~kg} / \mathrm{m}^{2}$. Her height was at $50^{\text {th }}$ to $75^{\text {th }}$ centile.

She is non-syndromic and has no neurodevelopmental concerns. The likely reason for obesity was monogenic and nutritional. As she showed more excessive weight gain for the proceeding one year, MRI brain and abdomen were done to exclude ROHHAD syndrome (Rapidonset Obesity, Hypothalamic dysfunction, Hypoventilation, and Autonomic Dysregulation) associated tumours, which were negative. Though we tried to tail off psychoactive drugs that also had adverse effects on appetite, our attempts failed with the child being more disturbed with her physical appearance

Her weight continued to increase, after discharge from the hospital, and so as the morbidity. She needed respiratory support throughout the day with ambulatory oxygen therapy during the daytime and BiPAP during sleep. Meanwhile, she was worrying immensely about her body image, which led us towards surgical options.

At a weight of $95 \mathrm{~kg}$ (BMI 45), she successfully underwent a laparoscopic sleeve gastrectomy at Colombo South Teaching Hospital. Nearly half of the stomach has been removed with the fundus; which easily stretches with food. Due to this small stomach tube, the amount of food ingested is restricted and she could take a small amount of food at a time thereby the amount of calories consumed is restricted which results in weight loss.

As the fundus of the stomach is removed the stomach becomes a "high pressure" tube after this procedure and some degree of reflux is seen in many patients. During the initial post-operative period and with some increased intake, she also had vomiting or intolerance of food. Now she takes small amounts of food at a time. To prevent her from getting micronutrient deficiency

She was commenced on vitamin B6, vitamin B12, Vitamin D 5000 IU daily. She was counselled regarding the volume and the composition of food. She had good compliance with the diet. Three months after the surgery, weight reduced by $25 \mathrm{~kg}$. Not only the weight but also her other parameters pre-op and 3 months post-op show a marked improvement. Total cholesterol dropping from 217 to $148 \mathrm{mg} / \mathrm{dL}$, fasting blood sugar from $104 \mathrm{mg} / \mathrm{dL}$ to $86 \mathrm{mg} / \mathrm{dL}$ and $\mathrm{HbA}_{1} \mathrm{C}$ from $7 \%$ to $6.3 \%$. Blood pressure which was $130 / 80 \mathrm{mmHg}$ before surgery is now around 100-95 / 60-65 mmHg. She came out of oxygen and respiratory support both during sleep and daytime.

She could overcome most of her psychological issues and start a better relationship with her sister while tailing off psychoactive medications and showing more interest in school work.

Currently, she is followed up at general paediatric, respiratory, endocrine and surgical clinics. She is being followed up for nutrition and growth, pubertal issues, dietary restrictions and respiratory function monitoring.

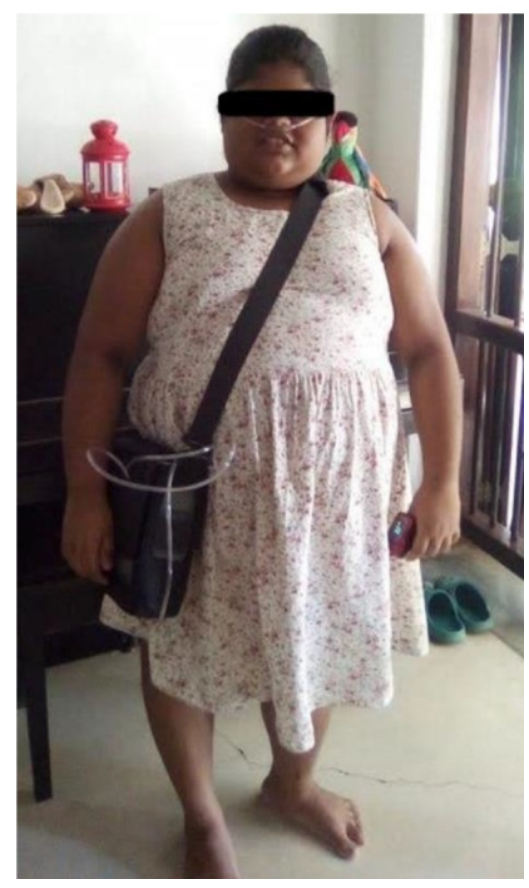

Figure 1: Before bariatric surgery 


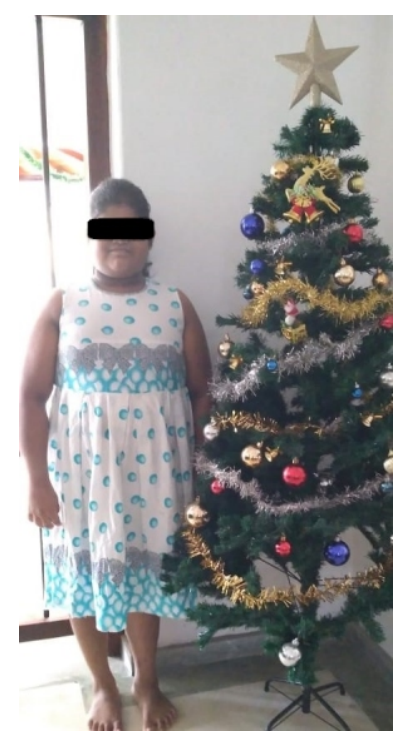

Figure 2: Three months after bariatric surgery

\section{Discussion}

Obesity is defined as weight for height more than three standard deviations above WHO growth standard median for children below five years of age and having a body mass index (BMI) $>+2$ SD for age and sex for children above five years. Obesity figures among children and adults are rising worldwide and its complications.

Obstructive sleep apnoea syndrome (OSAS) is a well-known complication associated with obesity. OSAS is the most important clinical entity within sleep-related breathing disorders, causing complete or partial upper airway obstruction leading to apnoea or hypoponea despite increased respiratory effort. Sixty percent $(60 \%)$ of obese children are affected by OSAS, and for every increment in BMI of $1 \mathrm{~kg} / \mathrm{m}^{2}$ beyond the mean BMI, the risk of OSA increases by $12 \%(6)$.

The possible mechanisms for OSAS among obese patients are the presence of fat at the level of the pharyngeal soft tissue reducing the calibre of the lumen and increasing the collapsibility of the structures, the increased fat content in the thoracic and abdominal walls affecting respiratory functions, adenotonsillar hypertrophy due to increased somatic growth, and increased critical airway closing pressure $(7,8)$.
Commonly used strategies such as adenoton-sillectomy and continuous positive airway pressure are less effective in obese children (9).

Lifestyle modification targeting weight reduction such as dietary modifications, increased physical activity, reduced screen time, and medical management of other comorbidities such as dyslipidaemia, hypertension and diabetes are our primary targets in managing obesity. It is questionable how practical this is and how long we can wait when children with morbid obesity are concerned. Their complications and quality of life worsen with time, leading to poor adherence to therapy. The failure of the above measures, and OSAS with oxygen dependency, made us seek surgical treatment, though there is not much evidence in the paediatric practice.

Bariatric surgery for morbidly obese children (BMI $>35 \mathrm{~kg} / \mathrm{m}^{2}$ ) with complications such as OSAS and cardiovascular morbidity is recommended, reducing the need for CPAP and other therapies (10).

A systematic review and meta-analysis of MS in adolescents done by Thenappan et al., demonstrated an average BMI difference from baseline to 1 year of $13.5 \mathrm{~kg} / \mathrm{m}^{2}$ after surgery. (11) Lifelong followup would be necessary for gastrointestinal complications and nutritional deficiencies (12). However, when managing children with obesity, encouragement and psychological support should be given throughout by the medical team involving the patient's family for better compliance.

\section{Conclusions}

Management of obesity needs consideration of the severity of obesity and associated complications. Medical and surgical options should be selected considering risk-benefit assessment. Thus obesityrelated comorbidity management requires a patient but sustainable multidisciplinary team approach with consistent encouragement and moral support to the patient.

(Informed written consent has been obtained from the child's parents to publish the report and photographs) 


\section{References}

1. Wittcopp C, Conroy R. Metabolic Syndrome in Children and Adolescents. Pediatr Rev. 2016 May; 37(5): 193-202. doi: 10.1542/pir.2014-0095. PMID: 27139327.

2. The IDF Consensus Definition of the Metabolic Syndrome in Children and Adolescents. International Diabetes Foundation. Accessed 4/1/2016 at http://www.idf.org/ webdata/docs/Mets_definition_children.pdf. (c) 2007, International Diabetes Foundation.

3. Cook S, Weitzman M, Auinger P, et al. Prevalence of a metabolic syndrome phenotype in adolescents: findings from the third National Health and Nutrition Examination Survey, 1988 - 1994. Arch Pediatr Adolesc Med. 2003; 157: 821-827. [PubMed] [Google Scholar]

4. Cook S, Auinger P, Li C, Ford ES. Metabolic syndrome rates in United States adolescents, from the National Health and Nutrition Examination Survey, 1999-2002. J Pediatr. 2008; 152: 165-170. [PubMed] [Google Scholar]

5. Wickramasinghe VP, Katulanda P, Seneviratne SN, Wijewickrama ES, Katulanda G, de Silva PH. Prevalence of obesity related metabolic abnormalities among 5-18year-old children: Preliminary data from the Western Province of Sri Lanka. In. $22^{\text {nd }}$ Annual Scientific Congress of Sri Lanka College of Paediatricians. Colombo: Sri Lanka, 2019 Aug (pp. 81-2).

6. Shwartz AR, Patil SP, Laffan AM, Polotsky V, Scheneider H, Smith PL. Obesity and obstructive sleep apnoea: pathogenic mechanisms and therapeutic approaches. Proceedings of the American Thoracic Society. 2008 Feb; 5(2): 185-192.
7. Dayyat E, Kheirandish-Gozal L, Capdevila OS, Maarafeya MM, Gozal D. Obstructive sleep apnoea in children: relative contributions of body mass index and adenotonsillar hypertrophy. Chest. 2009 Jul 1; 136(1): 137 144. doi: $10.1378 /$ chest.08-2568

8. Arens R, Muzumdar H. Childhood obesity and obstructive sleep apnea syndrome. Journal of Applied Physiology. 2010 Feb; 108(2): 436-444.

9. Kohler M. Risk factors and treatment for obstructive sleep apnea amongst obese children and adults. Current Opinion in Allergy and Clinical Immunology. 2009 Feb 1; 9(1): 4-9.

10. Dennis M. Styne, Silva A. Arslanian, Ellen L. Connor, Ismaa Sadaf Farooqi, M. Hassan Murad, Janet H. Silverstein, Jack A. Yanovski, Pediatric ObesityAssessment, Treatment, and Prevention: An Endocrine Society Clinical Practice Guideline, The Journal of Clinical Endocrinology \& Metabolism. 2017 March; 102(3): 709-757. doi: 10.1210/jc.2016-2573

11. Thenappan A, Nadler E. Bariatric Surgery in Children: Indications, Types, and Outcomes. Curr Gastroenterol Rep. 2019 Apr 25; 21(6):24. doi: 10.1007/s11894-0190691-8.

12. Beamish AJ, Reinehr T. Should bariatric surgery be performed in adolescents? European Journal of Endocrinology, 2017; 176(4), D1-D15. Retrieved Aug 27, 2021, from https:/eje.bioscientifica.com/view/journals/ eje/176/4/D1.xml 